\title{
Survival of the infected
}

The development of Kaposi sarcoma, a highly vascular tumour of endothelial cell origin, is associated with a herpes virus, KSHV (Kaposi sarcomaassociated herpesvirus). Individual KSHV genes have been shown to upregulate the phosphoinositide 3-kinase (PI3K)-Akt-mammalian target of rapamycin (mTOR, also known as FRAP1) pathway. Ling Wang and Blossom Damania have examined the role of this signalling pathway in endothelial cell survival and angiogenesis in response to the whole virus.

The authors generated KSHVinfected human umbilical vein endothelial cells (HUVECs) and found that KSHV infection activates the PI3K-Akt-mTOR pathway and inactivates a negative regulator of mTOR called AMPK. As this pathway is associated with cell survival, they examined the effect of KSHV on HUVEC apoptosis. KSHV-infected cells were resistant to apoptosis induced by starvation, etoposide and staurosporine, suggesting that KSHV infection aids cell survival.

To gain insight into how KSHV might influence endothelial cell angiogenesis, the ability of infected HUVECs to form tubular structures was assessed in vitro. KSHV-infected HUVECs showed increased cell survival and tubule maintenance in medium deprived of vascular endothelial growth factor (VEGF), an important factor in endothelial cell proliferation and angiogenesis. This finding supports studies suggesting that KSHV viral proteins continuously upregulate VEGF production and secretion in KSHV-infected endothelial cells, thereby leaving the infected cells resistant to exogenous depletion of VEGF. The ability of KSHV-infected HUVECs to form tubules was also increased by conditioned media from KSHV-positive B-cell lymphoma cells, further implying that KSHV-infected tumour cells secrete pro-angiogenic cytokines.

So, is KSHV-mediated activation of the PI3K-Akt-mTOR pathway important in endothelial cell tubule formation and angiogenesis? Chemical inhibition of mTOR, or activation of its inhibitor AMPK, completely disintegrated tubules formed by control HUVECs and reduced the number formed by KSHV-infected HUVECs. However, inhibition of PI3K had a modest effect on HUVEC tubule formation but killed the majority of KSHV-HUVECs, suggesting KSHVHUVECs are more sensitive to loss of PI3K activity.

This study reveals that KSHV infection confers a survival and angiogenic advantage to endothelial cells by activating the
PI3K-Akt-mTOR pathway, and suggests that therapies that inhibit PI3K activity might be beneficial for treating Kaposi sarcoma in the clinic.

\section{Katharine H. Wrighton}

ORIGINAL RESEARCH PAPER Wang, L. et al. Kaposi's sarcoma-associated herpesvirus confers a survival advantage to endothelial cells. Cancer Res. 68, 4640-4648 (2008)

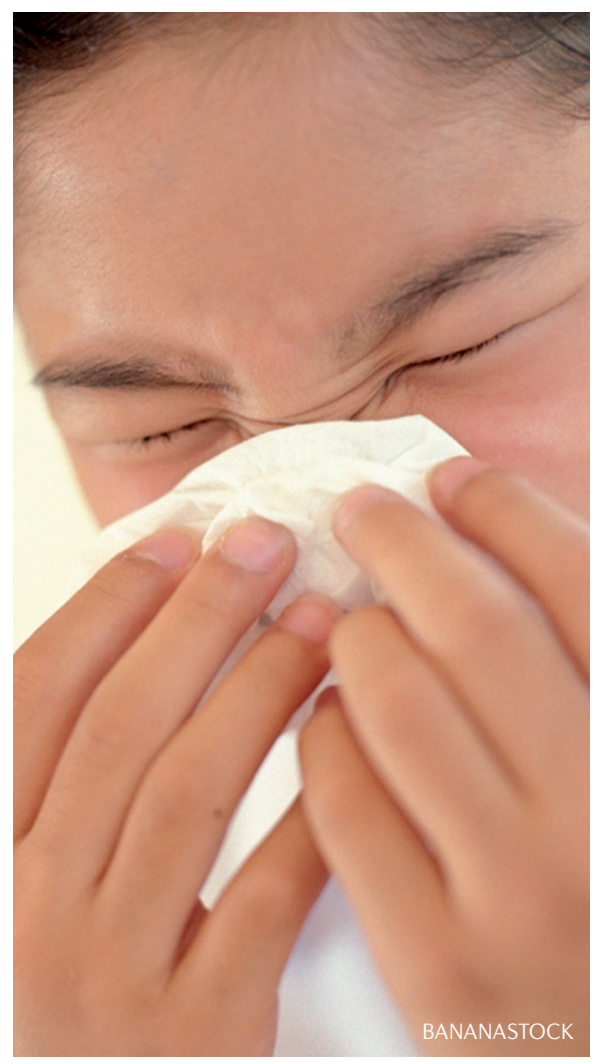

